\title{
The Fabrication of A MEMS-based Translational Vibratory Z-Axis Gyroscope Using DRIE on the Surface and Anisotropic Etching on the Backside of the Standard SOI Wafer
}

\author{
Tris Dewi Indraswati ${ }^{1,2}$, Adang Suwandi Ahmad ${ }^{2}{\text { Irman } \text { Idris }^{2} \text {, and Adrian Venema }}^{2}$ \\ ${ }^{1}$ Institut Teknologi Indonesia, Tangerang, Indonesia \\ ${ }^{1,2}$ Institut Teknologi Bandung, Bandung, Indonesia \\ tris.dewi@gmail.com, adangSahmad@yahoo.com, irman.idris@gmail.com, \\ adrinuha@bdg.centrin.net.id
}

\begin{abstract}
A new fabrication process of MEMS structure prototype of a translational vibratory z-axis gyroscope sensing element is presented. This structure consists of proof mass, driving devices, sensing devices, and suspensions, using the combination of MEMS bulk and surface technology with DRIE etching techniques on the surface and a suspended structure formation using anisotropic etching on the backside of the standard SOI wafer. The mask for the fabrication process was designed using L-Edit software. With this technique, the capacitance and the mass of proof mass can be increased thus increasing the gyroscope sensitivity to sense the Coriolis force. The MEMS-based translational vibratory z-axis gyroscope was static characterized by SEM measurements, visually and resistance measurement, wiring checking, and vibration checking by a given acoustic wave gyroscope excitation.
\end{abstract}

Keywords: MEMS-based translational vibratory z-axis gyroscope, coriolis force, MEMS technology, standard SOI wafer, angular movements.

\section{Introduction}

Gyroscope is a part of an inertial navigation system for detecting rotational movements in the vehicle to estimate position, velocity, and attitude rate of the vehicle. A gyroscope is used in various applications, for example in the field of vehicle stabilization, stabilization of moving equipment, consumer electronics, industrial, defense, until the medical field. For the development of new applications in the field of consumer electronics, medical equipment, prosthesis, spacecraft, and military applications, gyroscopes need to be lighter, smaller, and have a smaller power consumption. For miniaturizing the gyroscope dimension, gyroscope implemented with MEMS technology that is in the form of vibratory gyroscope.

The MEMS-based vibratory gyroscope system has three parts, namely the MEMS structure as sensing element, the driving and sensing circuits, and system's packaging. While the MEMS vibratory gyroscope sensing element has three parts, each of which is connected via a suspension, which is part of the actuator, the proof mass, and the sensor (Figure 1). Translational vibratory gyroscope sensing element is a central issue in the gyroscope design, as sensing element is a central part that couples energy from the primary, forced oscillations mode (actuation) into the secondary, sense oscillation mode (sensing).

It is of paramount importance that to be chosen vibratory gyroscope as a sensing device must be compact, rugged, reliable, with low power consumption, be mass fabricated and cheap in production costs. The sensor must have an unambiguousness in its performance and a reliability in the fulfilment of its task as a sensor. The measured data - representing the movements of the vehicle must be of the highest degree of accuracy possible - are wireless transmitted to a ground station. The operation of the sensor must withstand bad weather conditions and engine vibration as good as possible.

MEMS-based translational vibratory gyroscopes sensing element can be implemented with

Received: October $4^{\text {th }}, 2013$. Accepted: December $16^{\text {th }}, 2013$ 
various micromachining technology, among others, bulk micromachining technology, surface micromachining technology, or integration between bulk and surface micromachining technology. With the integration of both micromachining technologies, bulk and surface micromachining technology, the gyroscope will have larger mass from the bulk micromachining technology and the conditioning and processing circuits can be integrated in the gyroscope chip, thereby reducing its parasitic capacitances.

Currently, there are several techniques developed to combine the bulk and surface micromachining technology, such as by the deep-reactive-ion-etch (DRIE) CMOS-MEMS [4] [18] [19] [20], the use of SOI wafers as the gyroscope substrate [5] [21], and the use of multilayered metal or polysilicon as capacitances in combdrives for actuators and sensors.

In the technique developed, there are some drawbacks such as the curl out of plane and relatively small capacitance problems from the use of multi-layered metal [4] [8] [9]. While the technique of using SOI wafers, is used specifically for micromachining SOI wafer with thick enough silicon dioxide layer (about $4 \mu \mathrm{m}$ ) which are quite expensive [5]. While the techniques used are also still relies on making release hole to release the sacrificial layer so that the magnitude of the obtained mass of the proof mass is not optimal. While in the DRIE CMOSMEMS process, curl out of plane problems can be solved, however, there is still a relatively small capacitance problem, because it still uses multi-layered metal as an actuator/sensing capacitance [4].

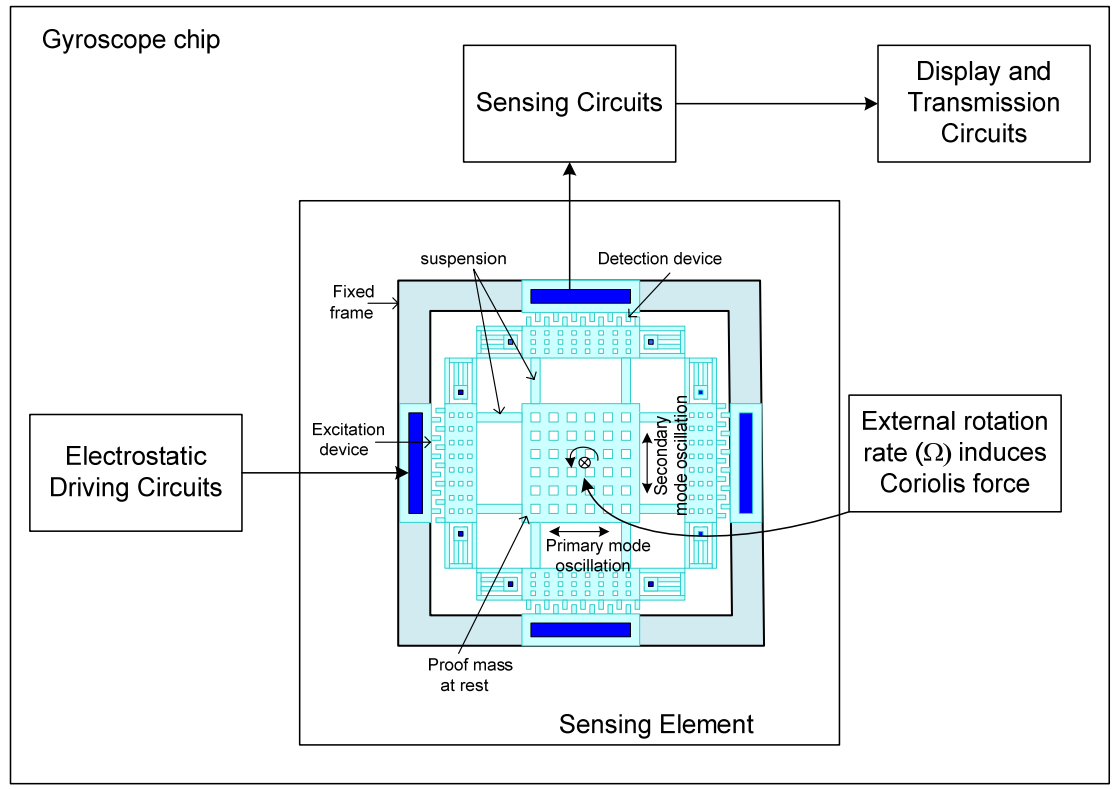

Figure 1. MEMS-based vibratory gyroscope chip block diagram.

In this research, the exploration of gyroscope fabrication techniques is conducted. The proposed fabrication technique is the use of standard SOI wafers as substrates and integrating the bulk and surface micromachining technology with DRIE process on the surface and anisotropic etching on the back of the wafer. With this technique, the release hole needed for sacrificial layer releasing can be removed and a greater mass will be obtained, so it will be more sensitive to the Coriolis force. Besides the gyroscope fabrication process become simpler, the price of standard SOI wafer cheaper than micromachined specific SOI wafer. Thus it can be expected that the product sensor compact, rugged, reliably, with low power consumption, be mass fabricated and cheap in production costs. 


\section{The Important Aspects of Silicon Micromachining Fabrication Technology}

In the manufacture of MEMS structures, there are several aspects that must be considered. Patterns transferred in the photolithography process, the etching process for a moving mechanical structure formation, and the selection of materials forming the structure, all of which are important aspects in the fabrication of MEMS devices.

\section{A. Photolithography Process}

Photolithography process is a pattern transferred process from the mask on the glass plate into the wafer substrate, which is done by deposit the light-sensitive material (photoresist) onto the wafer and provide the appropriate light exposure, then develop the resist to get the pattern, etch the layer, and stripping the resist remain. Photolithography will define the shape of the MEMS device structures (gap between parts of the structure and the width of the structure).

Pattern transfer in the formation of the MEMS structure is important, because the aspect ratio - the ratio between the width of the surface structure to a depth of etching to be done determine how thick the resist to be deposited onto wafer and how much energy should be provided to exposure the resist. The differences in resist thickness can be achieved by changing the spinner rate when coating the resist onto wafer, set the resist volume by set the number of drops, and set the coating time. While the differences in the exposure power dose at the exposure stepper machine will determine the adhesion between the resist and the wafer for holding the etching until at a certain depth. The resist thickness and the adhesion power of resist - wafer will determine the resolution error in the pattern transfer process. The more fitting recipe formulated the resolution error will also shrink. The appropriate photolithography prescription formulation must be done by performing several experiments.

The thickness and uniformity of the layer that forms the structure can also cause failures in the patterns transfer process, can be either resolution error or error due to the misalignment of the devices structural patterns.

\section{B. Etching Process}

Etching process is a fundamental process used in microelectronics and MEMS to engrave a pattern on the material. Etching is widely used in material processing to draw the pattern, eliminating the surface damage and contamination, and fabricate the three-dimensional structures.

Since many etching procedures have to be performed when forming the MEMS structure, it is important to choose beforehand the correct crystal orientation for the silicon material; $<100>$ or $<111>$. The external shape of the microstructure body, cannot be arbitrarily chosen, but are necessarily determined by the crystal morphology.

Considerations for the selection of special etching process include: 1. Etching rate; 2. Uniformity; 3. Selectivity, it is important to select the proper mask to be used in the etching process and the material film to stop the etch process; 4 . Directionality: etching can be isotropic (omnidirectional) or anisotropic (directional).

By using etchant with high selectivity to different crystal orientation, the desired shape and dimensions of the cavity can be predicted and fabricated in highly reproducible manner. Such etching can be applied also to the back of the wafer to reduce the thickness of the silicon substrate locally, to form a silicon membrane with a certain thickness [30]

\section{B.1. DRIE Etching}

Reactive Ion Etching (RIE), also known as plasma etching or dry etching, and the development of RIE, i.e. Deep Reactive Ion Etching (DRIE) is a process that combines physical and chemical effects to remove material from the wafer surface. The most important advantages of RIE etching to wet etching is its ability to perform directional (anisotropic) etching regardless of the plane of crystal material.

Plasma etching process variables (such as the Radio Frequency (RF), pressure, gas etcher flow rate, and temperature) can be varied to optimize the etch rate, selectivity, sidewalls angle, 
or other responses. The key processes of DRIE process are: etching rate, anisotropy/sidewalls angle, mask selectivity, substrate selectivity, etched surface quality, and throughput.

The main DRIE techniques are the Bosch and cryo-process techniques [18].

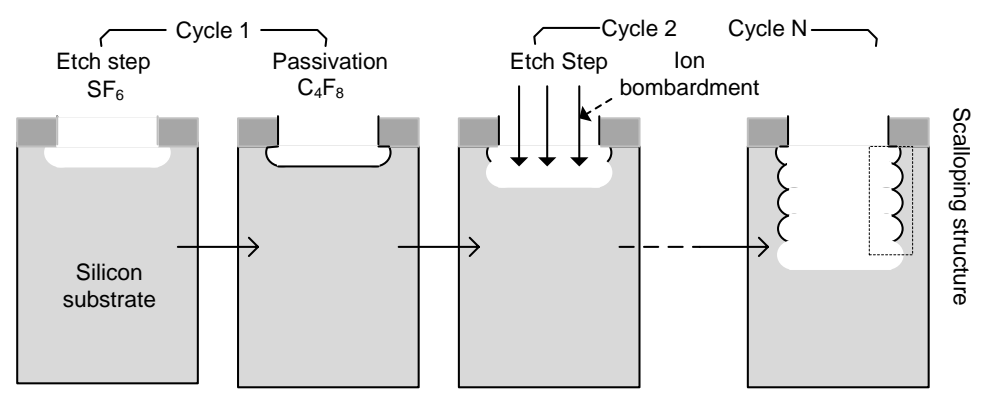

Figure 2. Schematic drawing of DRIE process sequence in Bosch process[18]

Bosch process involves a sequence of repeating cycles as shown in figure 2 [18]. Each cycle consists of an etching step immediately followed by a passivation step. During the etching step, $\mathrm{SF}_{6}$ is used to etch the silicon isotropically, and this creates a small cavity on the silicon surface. In the passivation step, the entire exposed surface is covered with a thin polymer layer, which is given by octofluorocylobutane $\mathrm{C}_{4} \mathrm{~F}_{8}$. This polymer layer is inert to chemical etching of $\mathrm{SF}_{6}$, but can be removed by physical bombardment of ions. Thus, in the beginning of etching step in the next cycle, the protection layer at the bottom of the cavity, which faces the bombardment of plasma ions, is eliminated, while the protective polymer on the sidewalls of the cavity remains. Therefore, after the next cycle, another small cavity is formed under the previous cavity. By repeats the cycle many times, a deep trench can be etched into the silicon substrate. The time period of each cycle is only a few seconds, and therefore, only a thin layer of silicon (several hundred nanometres) is etched in every cycle. This process results in a smooth surface with a periodical and slight scalloping (only a few tens of nanometres) on the sidewalls. In general, the process creates a vertical trenches into the silicon substrate, and the angle of the trench sidewalls can be somewhat controlled by tuning the balance between the etching and passivation phases. This can be achieved by modifying the plasma conditions during the two steps. By using this Bosch technique, etching process turns an isotropic etching into a vertical anisotropic one.

\section{B.2. Material Composing MEMS Structure}

Material selection in the microsystems is determined from the microfabrication limitations. MEMS device performance depends on the material properties build the device. Most of these materials are used in the form of thin layers. Due to the submicron to $\mathrm{mm}$ size scale of the MEMS devices, their material properties can substantially differ from the ones found in bulk material. Even not inherently scale-dependent, properties like density and Young's modulus, can be altered from bulk values by creation of non-equilibrium structures. Owing to the small size and extreme aspect ratio of MEMS devices, new failure mechanism, such as crack-related and surface effects can play a greater role. Environmental effects, such as adsorption / desorption induced surface stress, can change the mechanical, electrical, and resonant properties of the suspensions and proof mass [24].

Materials build the MEMS structure of the vibratory gyroscope sensing element will determine the sequence of the process of the gyroscopes fabrication; determine the aspect ratio (the ratio between the minimum width to the height of the structures) that can be implemented; determine the MEMS structures properties such as curl out of plane, residual stress, and how much spring / suspension constantan. This spring constantan will determine the drive frequency of the MEMS gyroscope structures, and in turn will determine the magnitude of the Coriolis force can be sensed by the gyroscope. 


\section{State of The Art of The Mems-Based Translational Vibratory Gyroscope}

To find a fabrication process that can effectively and efficiently to create translational vibratory MEMS gyroscopes, the evaluation of the current fabrication process has been done. This evaluation involves the assessment of a number of factors, among others [28]: the critical dimension (smallest dimension that can be manufactured), the precision of the process (i.e., the dimensional accuracy or nominal device dimension), materials available for fabrication, assembly requirements to produce functional devices, process scalability (i.e. can enlarge the producible devices quantity), and integration with other fabrication processes (e.g. microelectronics).

Here are presented some of the latest technology in the fabrication process of MEMS-based translational vibratory gyroscope.

\section{A. Bulk and Surface Integration Micromachining Technology}

Initially, there are two kinds of silicon-based micromachining technology, namely surface micromachining technology and bulk micromachining technology. Each has advantages and disadvantages. Integrating the two technologies to fabricate the MEMS gyroscope, results in several advantages [25], among others, the gyroscope will have larger mass and the electronic circuits can be integrated in one chip, thereby reducing its parasitic capacitance. These will increase the sensitivity of the gyroscope to the Coriolis force. The parasitic capacitances must be taken into account here, because the gyroscope use the combdrives as capacitances, as the devices to actuate and sense, and it should be remembered that the order of the capacitance in gyroscope are tens to hundreds femtoFarad and the order of changes resulting from the sensing are attoFarad to femtoFarad.

In the integration of the surface and bulk micromachining fabrication process, the fabrication of CMOS-based signal processing and conditioning circuits are integrated in a single wafer with the MEMS structure of the MEMS-based vibratory gyroscope sensing element. Thus this technology is called CMOS-MEMS technology.

In the surface CMOS-MEMS fabrication process, there is a crucial problem to be solved, that is curl out of plane - a bending out the plane - which can reduce the sensing accuracy due to the sensed capacitance obtained is not optimum as designed, even the mass may not move in plane again. This problem is due to the different residual stress of materials composing composite microstructure. It produces non-symmetric stress gradient in the microstructure. At the sacrificial releasing etch, the structure tends to warp out of the device plane. Figure 3 shows the curl out of plane problems that occur. Besides the different residual stress, this problem can also due to the material used is too thin and have high stress constant. To overcome this problem, the following method is proposed [7], among others, use a thicker layer, using materials that have a low stress constant, the process modification yields a more uniform structure, the higher material density of mass, so the mass is larger, and designing the frame of structure for the counterweight of curl out-of-plane.

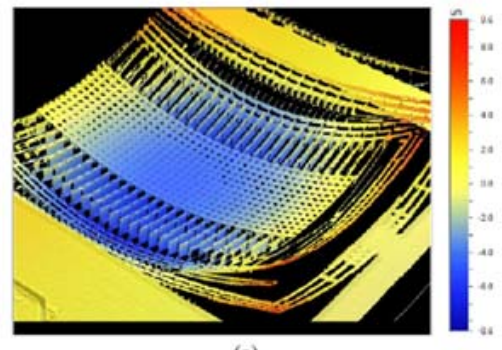

(a)

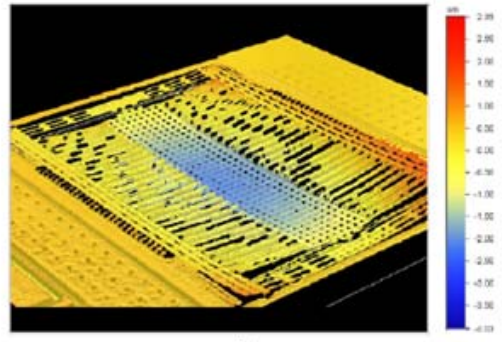

(b)

Curl comparison. (a) Aluminum gyroscope. (b) Copper gyroscope.

Figure 3. Curl out of plane problem comparation in CMOS-MEMS gyroscope, between the gyroscope using multi-layered aluminium and multi-layered copper[7]. 
In the bulk CMOS-MEMS fabrication process, MEMS structures are still using multilayered metal to build combdrives as actuation and sensing capacitance. The mass of the proof mass become greater due to addition of a part of bulk as the supporting structure of multilayered metal stack which serves as actuator and sensing devices. But not so with the capacitance, because the capacitance values obtained from surface overlapping of the cross section of the multi-layered metal. The metal forms the multi-layered metal also determines the magnitude of the aspect ratio can be obtained from the fabrication process, while large aspect ratio determines the magnitude of the capacitance can be obtained from the structure.

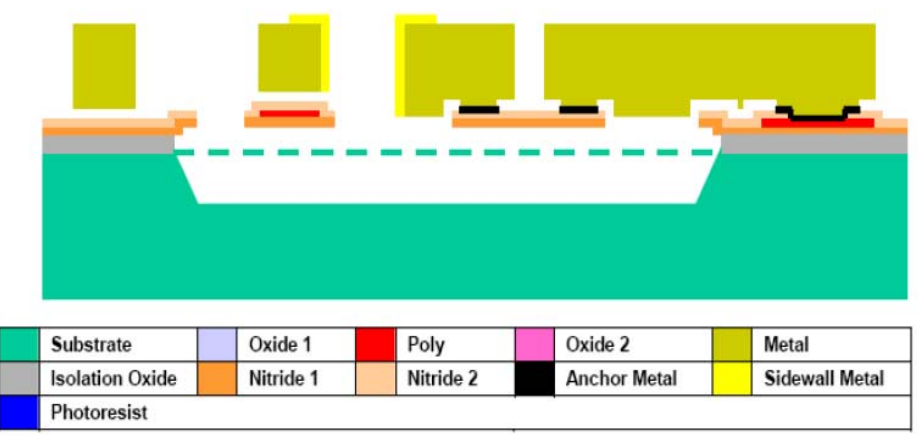

Figure 4. Fabrication process flow with MetalMUMPS technology[38]

Two double polished wafers were used in the MetalMUMPS fabrication process (figure 4). The movable structure was made on one side of the first wafer, while the cavity structure as vast the movable structure, was made on the surface of the second wafer. After bonding process of the two wafer, the movable structure can be found hanging on the prepared cavity.

\section{B. Silicon - on - Insulator (SOI) Wafer - Based MEMS Translational Vibratory Gyroscope Fabrication}

A silicon - on - insulator (SOI) wafer is the perfect material to be used in bulk micromachining. The layer for building the devices is located above the insulator layer. By patterning the silicon layer and the oxide layer beneath, a free - standing structure can be made, which is electrically isolated and mechanically anchored.

Silicon - on - Insulator (SOI) wafer - based MEMS translational vibratory gyroscope fabrication process can be performed in some ways, namely with the surface, bulk, SOIMUMPS, and a combination of surface and wafer bonding SOI fabrication process. In the surface SOI gyroscope fabrication process, the gyroscope characteristics similar to the gyroscope fabricated using single crystal silicon-based surface micromachining technology. The small release holes must be made to make the suspended structure perfectly release from the substrate. The existence of this release holes can reduce the mass should be obtained, thereby reducing the sensitivity of the gyroscope to the Coriolis force. The thickness of the oxide should support the establishment of the suspended structure, also ensure that no stiction between the suspended structure with the substrate; this is at least 1 micron oxide thickness for the surface micromachining process.

The one mask bulk SOI fabrication process [5] is almost equal to the surface SOI micromachining process. Although it only uses one mask, but this technology still requires release holes that can reduce the mass of the proof mass, which ultimately reduces the sensitivity of the gyroscope. This problem can be solved by making the thickness of the silicon structure thicker, which allowed with the DRIE etching process, so the mass becomes larger. Nevertheless, for releasing the suspended structure, it requires a minimum oxide thickness of 2 microns and significant release hole dimensions. 
The SOIMUMPS fabrication process uses specific SOI wafers for micromachining process with minimum oxide thickness of 1 micron. SOI wafers used here have 10 microns silicon thickness over the insulator (oxide) and 400 microns thick silicon handling. In particular, the proof mass of the gyroscope does not require release holes, because anisotropic etching can be done from the back of the wafer to make the suspended structure.

The bulk-SOI micromachining fabrication process can be integrated with vacuum - anodic - bonding process. The suspended structure can be obtained after SOI wafers bonded with another wafer, so it needs more than one wafer.

\section{The Fabrication of Mems-Based Translational Vibratory Gyroscope Base on Standard Silicon-on-Insulator (Soi) Wafer Using Drie Etching Processes on The Surface and Anisotropic Etching on The Back of The SOI Wafer}

In this research, MEMS-based translational vibratory gyroscope was fabricated using a standard SOI wafer with DRIE etching processes on the surface and anisotropic etching on the back of the SOI wafer. This technology is similar to the SOIMUMPS technology, but it uses standard SOI wafer with $400 \mathrm{~nm}$ oxide thickness as an insulator, rather than specific SOI wafers for micromachining process.

Vibratory gyroscope fabrication process conducted in DIMES TU Delft is different from previous processes conducted by other researchers. Huikai Xie from University of Georgia USA made the vibratory gyroscope using the integration of surface and bulk micromachining technology. Xie built the multi-layered of aluminium as the capacitances on the silicon surface and perform DRIE etching on the back to form a suspended mass-spring system. The wafer used is single crystal silicon. The advantages mentioned here is the suspended proof mass does not require release holes. By Xie processing method, the gyroscope with vertical actuation can also be made from multi-layered aluminium structure. Multi-layered aluminium structure can be replaced with another metal, so that the curl out-of-plane properties can be modified.

While another researcher, Cenk Acar, used SOI wafer with 100 micron thick epitaxial layers to form a mass-spring system[5]. However, Cenk Acar still used proof mass with release holes approach and releasing etch from the surface to built the suspended mass-spring system structures, using 4 microns sacrificial oxide layer thickness. The gyroscope work based on translational vibration of the suspended mass-spring system structures.

With SOIMUMPS technology, gyroscope fabrication is performed on the specific SOI wafers for micromachining process, with the minimum oxide sacrificial layer of 1 micron.
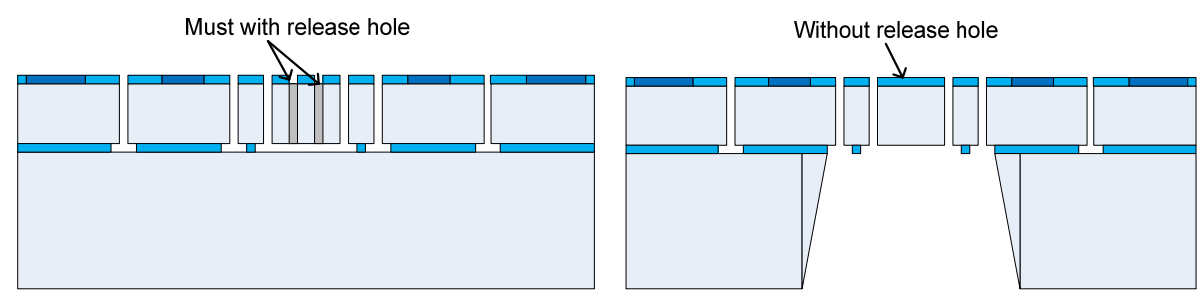

Figure 5. Gyroscope sensitivity increase with the increasing silicon thickness via epitaxial layer in SOI processing and enlarge the gyroscope proof mass by processing of sacrificial releasing etch without release holes.

While the new fabrication method done in DIMES is a combination of DRIE at the surface - standard SOI - anisotropic backside etching. Advantages of this method include:

- The mass of the sensing element (proof mass) can be heavier, because it can be made without release holes, by releasing etching from the backside (figure 5), and setting the epitaxial layer thickness of the silicon structure layer. DRIE etching on the surface allows the formation of a relatively sharp structure for the depths of up to more than 100 microns, so enabling to manufacture the structures with high aspect ratio. The large proof mass 
Tris Dewi Indraswati, et al.

closely related with the gyroscope sensitivity to the sensed Coriolis force when there is an external angular rate.

- The large aspect ratio allows for getting the large enough actuation and readout capacitance due to larger overlap parallel plate area and a smaller gap.

\section{A. Process Flow}

MEMS-based translational vibratory gyroscope fabrication in this research is implemented with two kinds of process sequence, each of which requires a 3 mask and 4 mask, using SOI wafer with an epitaxial layer of high conductance single crystal silicon layer.

MEMS-based translational vibratory gyroscope fabrication process flow is shown in Figure 6.
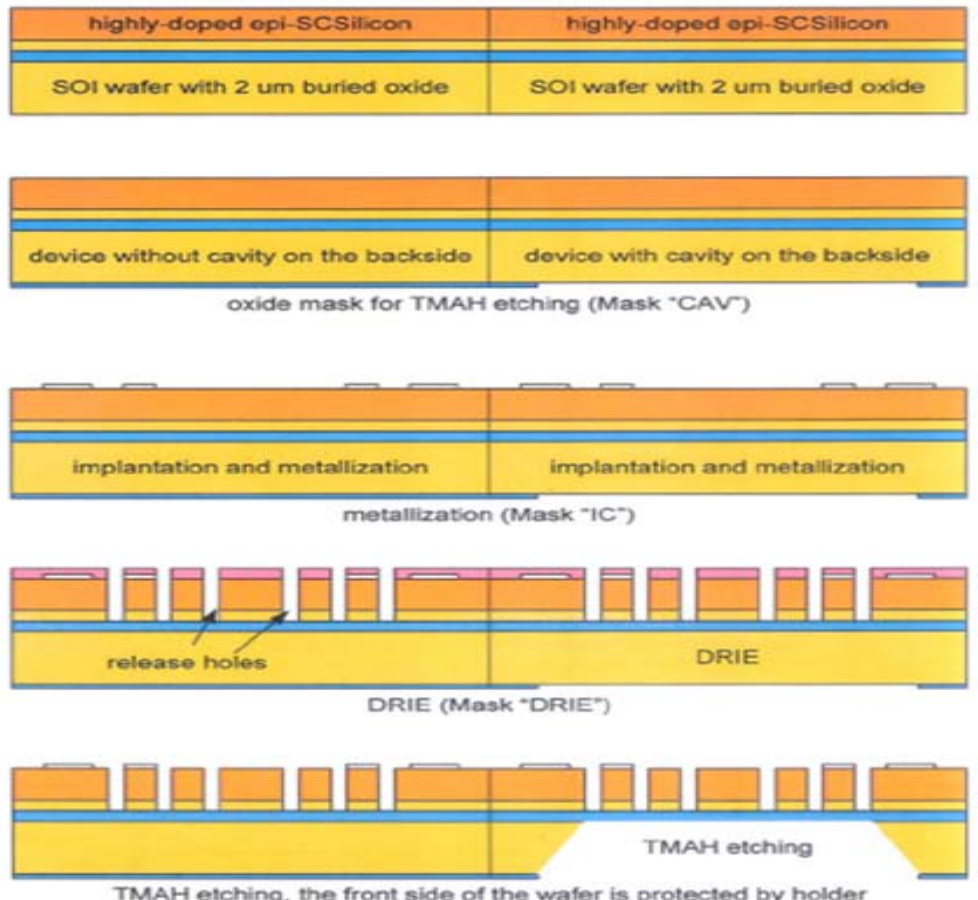

TMAH etching, the front side of the wafer is protected by holder

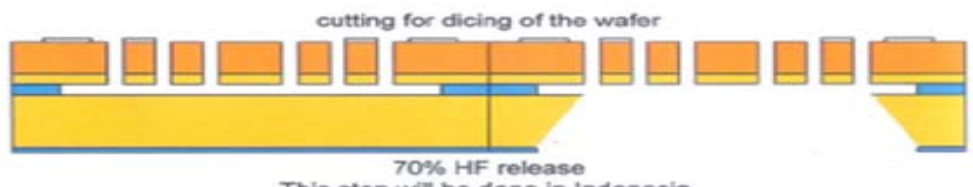

a.

b.

Figure 6. Gyroscope process flow with 3 masks

\section{B. Layout and Mask}

Layout is designed to be implemented on $10 \mathrm{~mm} \times 10 \mathrm{~mm}$ chip frame size. In this $10 \mathrm{~mm} \mathrm{x}$ $10 \mathrm{~mm}$ chip frame, 6 different layouts can be drawn with the same type of mask, to be elaborated, by the division of chip size of $5 \mathrm{~mm} \times 3.3 \mathrm{~mm}$ (Figure 7), including the test pattern.

When drawing the MEMS gyroscope sensing element layout, there are several things that must be considered in order the gyroscope can work properly, among other :

- The anchors dimensions and distance to the sidewall of suspended structure must be taken into account, so the anchors will not be etched when sacrificial layer releasing etch is done (Figure 8). In this case, the anchor width ( $\left.\mathrm{W}_{\mathrm{a}}\right)$ must be greater than $25 \mu \mathrm{m}$ (the width that can be erased when isotropic etching is done - note from all directions, left and right) 
- The space between the release holes should be taken into account in order the sacrificial layer can be perfectly etched (there is an effect of aspect ratio dependent etching (ARDE)) when sacrificial layer releasing etch is performed (Figure 9).

- The metal pattern must be smaller than the DRIE pattern so that when DRIE etching is done, no dirt that falls on an improper place.

- Parasitic capacitance at the metal pad size of $200 \mathrm{um}$ x $200 \mathrm{um}$ is about $1 \mathrm{pF}$, and it will be the device parasitic capacitance. Thus it should be considered because it may cause noise approximately $25 \mathrm{x}$ the signal value. Designers should calculate device signal to noise ratio (SNR) to produce accurate measurements. This is also applied to the pattern of interconnecting parts of sensing elements with metal pad. Parasitic capacitances due to the enlargement of the pattern of pad and interconnection section sensing element with pad should be taken into account, because it could be a larger capacitance than the capacitance to be measured. (approximately10 $\mathrm{pF}$ (parasitic) compared with $250 \mathrm{fF}=0.25 \mathrm{pF}$ (sensing)).

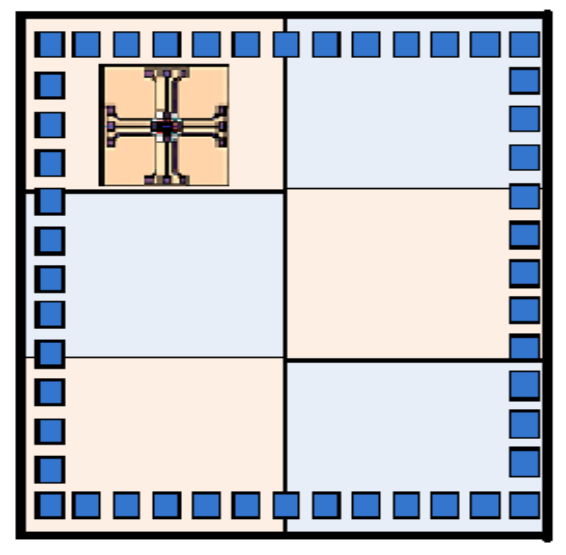

Figure 7. Gyroscope layout in the chip frame $10 \mathrm{~mm}$ x $10 \mathrm{~mm}$ in size, with 6 types of layout, each drawn in chip $5 \mathrm{~mm} \times 3.3 \mathrm{~mm}$ in size.

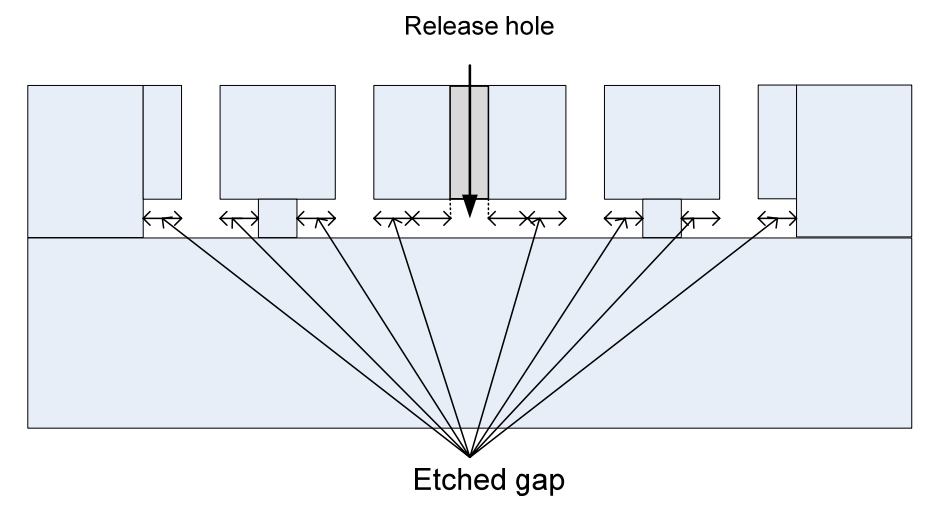

Figure 8. Anchor dimensions and space from sidewalls of the suspended structure must be taken into account in order it will not to be etched when sacrificial layer releasing etch is performed. 
Tris Dewi Indraswati, et al.

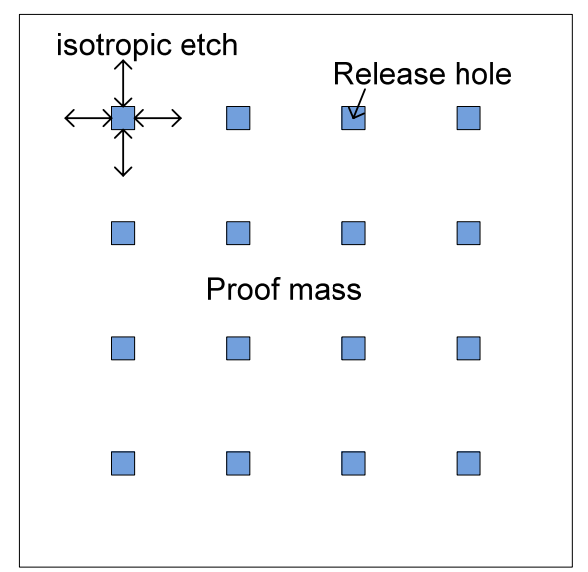

Figure 9. The space between the release holes should be taken into account in order the sacrificial layer can be perfectly etched when sacrificial layer releasing etch is performed.

\section{Masks Pattern for Photolithography}

The following is the mask layout for fabrication of the MEMS-based translational vibratory Z-axis gyroscope using SOI wafer (figure 10). MEMS-based translational vibratory Z-axis gyroscope is fabricated using 3 masks : gyroscope with release holes that sacrificial layer releasing etch is performed from the frontside and from the backside of wafer, and gyroscope without release holes that sacrificial layer releasing etch is performed from the backside of wafer, the three gyroscope is made without an insulation layer, and using 4 masks : gyroscope without release holes that sacrificial layer releasing etch is performed from the backside of wafer and it has an insulation layer.

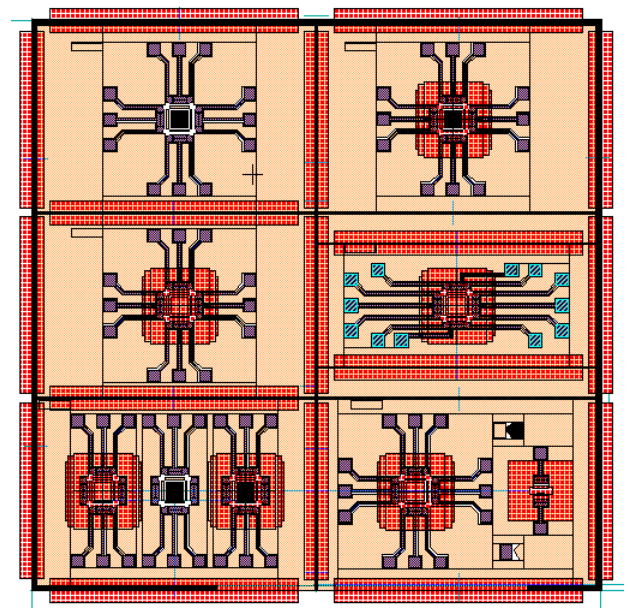

Figure 10. Chip 10mm x $10 \mathrm{~mm}$ of the MEMS-based vibratory gyroscope, consists of 6-pattern layout : 2 gyroscope with proof mass that have the releasing holes - one uses front opening (sacrificial layer releasing from the front of wafer) and one uses back opening (sacrificial layer releasing form the backside of the wafer); 2 gyroscope with proof mass without the release holes - one without insulation layer above the metal layer and another one with an insulation layer above the metal layer; and test pattern for combdrive when drived, gyroscope with different frequency, test pattern for Young modulus, and test pattern for residual stress.

Once the file .tdb in L-Edit has been completed, then the .tdb file is converted into the GDSII files form. Files in this form will be translated by mask fabrication machine as the mask 
to be made. Figure 11 is an image of the mask pattern that has been converted in the GDSII files form to sent to the mask fabrication machine.

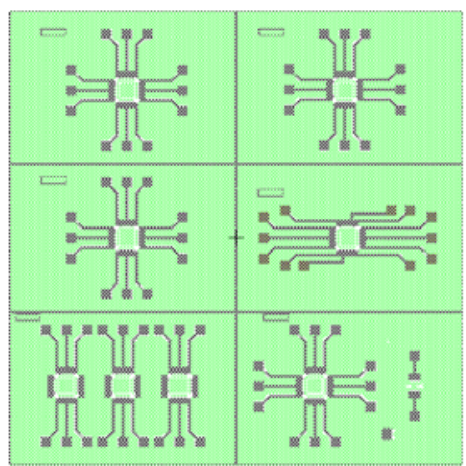

Figure 11. Mask pattern changes image after .tdb file in L-Edit had been converted to the GDSII file to send to the stepper or contact aligner fabrication mask machine.

MEMS vibratory gyroscope implementation is made for the gyroscope with the natural frequency of $10.7 \mathrm{kHz}$ and $8.3 \mathrm{kHz}$. Drive quality factor is 7700-61700. Sensing bandwidth between $560-605 \mathrm{~Hz}$ with a quality factor of 91-113.

Figure 12 and Figure 13 show an example of the mask pattern that has been translated by CONTACT ALIGNER and STEPPER machine into a mask form to be fabricated. Only the mask of the pattern of cavity formation at the backside of wafer that used for sacrificial layer releasing that is fabricated using a STEPPER machine (Figure 26). The rest mask is fabricated using the CONTACT ALIGNER machine.

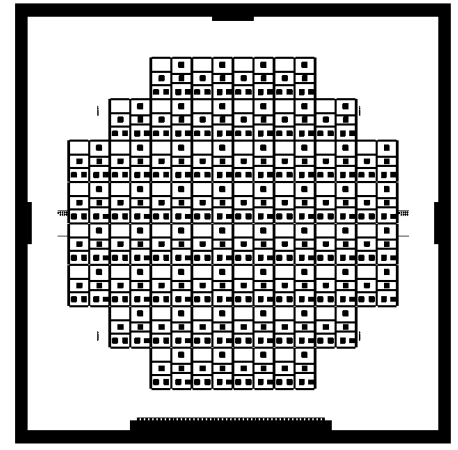

Figure 12. Mask pattern for $\mathrm{KOH}$ etch to make the gyroscope cavity at the backside of the wafer for sacrificial release process.
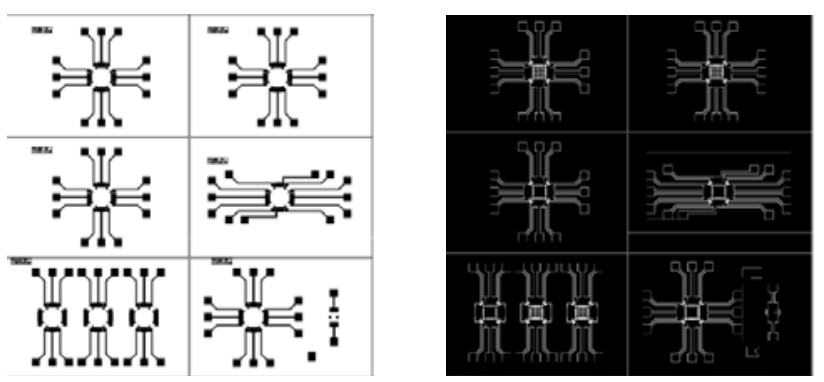

Figure 13. Mask pattern for metal interconnection formation and DRIE, respectively 


\section{Dicing and Packaging}

Dicing (cutting wafers into the small chips) is done by designing cutting lines in the "CAV" (DRIE step) mask (see figure 14), so V-groove cutting lines can be made on $\mathrm{KOH}$ step (opening/structure releasing step from the backside of wafer, in this case, there is an alternative for etchant using TMAH). Thus dicing can be done manually. The width of the grooving pattern on the backside should be taken into account and adjusted to the crystal orientation of the wafer in order to get V-groove with the depth of approximately $100 \mu \mathrm{m}$ in etching pattern with $\mathrm{KOH}$ or TMAH. While the packaging is performed with available chip package and only the gyroscope sensing element are packaged, without the signal processing circuit.

\section{Results and Analysis of The Mems-Based Translational Vibratory Gyroscope Fabrication}

The following are the results obtained from the fabrication of MEMS translational vibratory gyroscopes and their analysis. The analysis is performed by means of static characterization of the gyroscope, i.e. measurement, observation and testing when the gyroscope at rest or in the absence of external excitation.

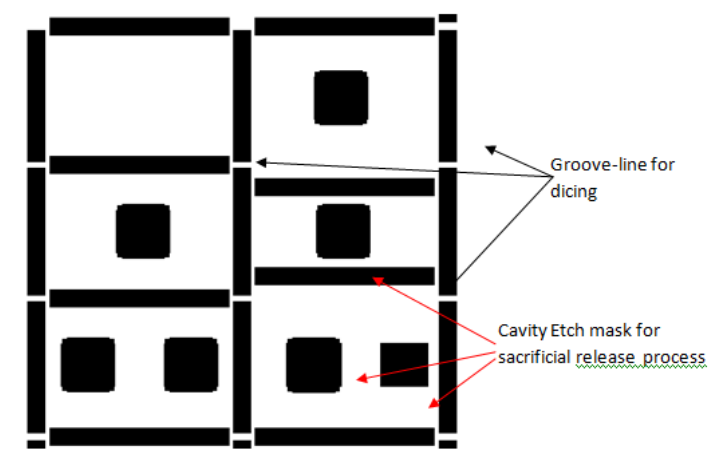

Figure 14. Mask pattern for cavity etch needed for sacrificial release process and grooving lines for dicing process.

This characterization includes measurements of physical geometry of MEMS gyroscopes after the implementation (static deflection without input) using SEM, performs physical checks on the gyroscope which includes visually physical form, wiring checking on the already packaged gyroscope, stiction problems checking on the gyroscope after fabrication to ensure that the masses are suspended not sticky on the substrate, and perform the vibration testing to test whether the mass-spring system in gyroscope can work well.

\section{A. Visual Observation and Physical Measurements of the MEMS-Based Translational Vibratory Z-Axis Gyroscope using SEM}

Geometry physical measurement of the MEMS-based translational vibratory Z-axis gyroscope is performed directly during the gyroscope fabrication in DIMES, Delft University of Technology, Netherlands. Checks are conducted regularly on the important fabrication steps and when the gyroscope fabrication process has been completed. The measurement process is carried out to evaluate the fabrication process, the fabrication recipes evaluation, and also for final check of the gyroscope geometry dimensions to be compared with the designed dimensions.

Discussion of some important results obtained from visual observations and physical measurements with SEM machine will be viewed in terms of the fabrication process evaluation, fabrication failure evaluation, visually checking of the physical gyroscope, geometry dimension measurement. Here is the discussion. 


\section{SEM Image for Fabrication Process Evaluation}

The important fabrication step process evaluation for the gyroscope fabrication is when DRIE etching done on the surface of the SOI wafer. The recipe for DRIE etching step should be re-evaluated when the results geometry fabrication process obtained does not comply with the acceptable tolerance of the designed value.

Figure 15 shows the difference in the width dimension of comb drive fingers results from DRIE etching with the first prescription.

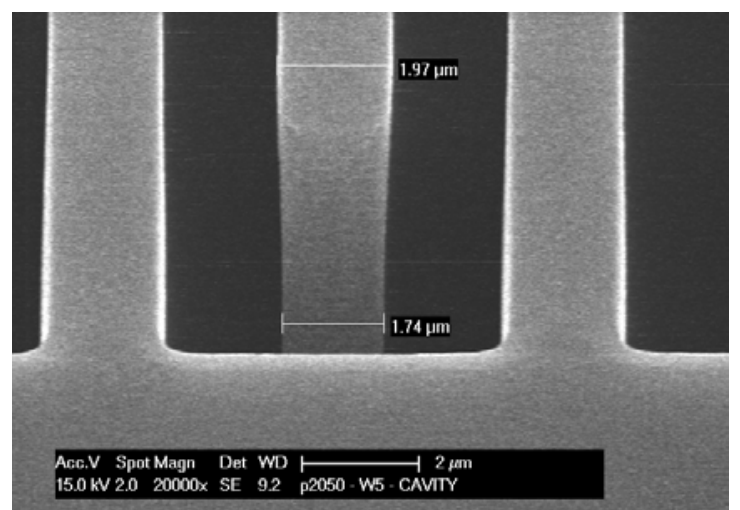

Figure 15. The difference of the dimension of combdrive fingers width result from the DRIE etch with the first recipe.

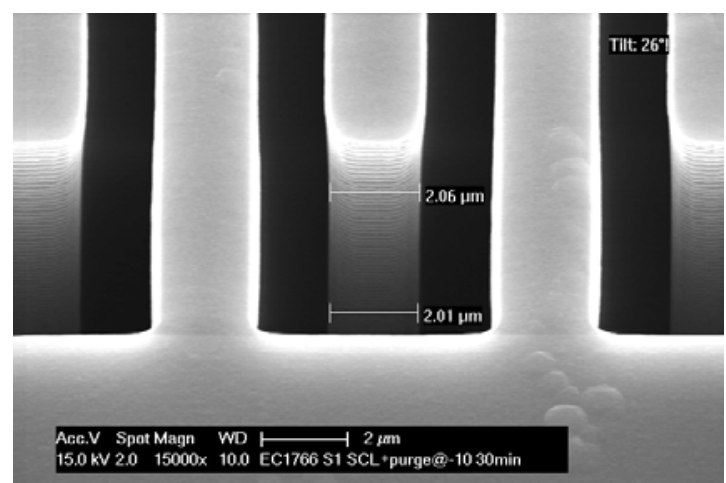

Figure 16. The dimension of comb drive fingers result from the modification of DRIE etch recipe.

Figure 15 shows the size of comb finger dimension obtained is $1.97 \mu \mathrm{m}$ at the top and 1.74 $\mu \mathrm{m}$ at the bottom. Where as the expected size was $2 \mu \mathrm{m}$. It means that there are different dimensions of approximately [((2-1.97) / 2) x 100\%] or $1.5 \%$ of which should be at the top, and $[((2-1.74) / 2) \times 100 \%]$ or $13 \%$ of the supposed at the bottom. Therefore, the recipe is modified, so finally the final dimensions of the comb fingers obtained are $2.06 \mu \mathrm{m}$ at the top and $2.01 \mu \mathrm{m}$ at the bottom (Fig. 16) or 3\% of which should be at the top, and $0.5 \%$ of which should be at the bottom. However, there is variation in the dimension of the process results, although it is performed on the same batch (Figure 17). 
Tris Dewi Indraswati, et al.

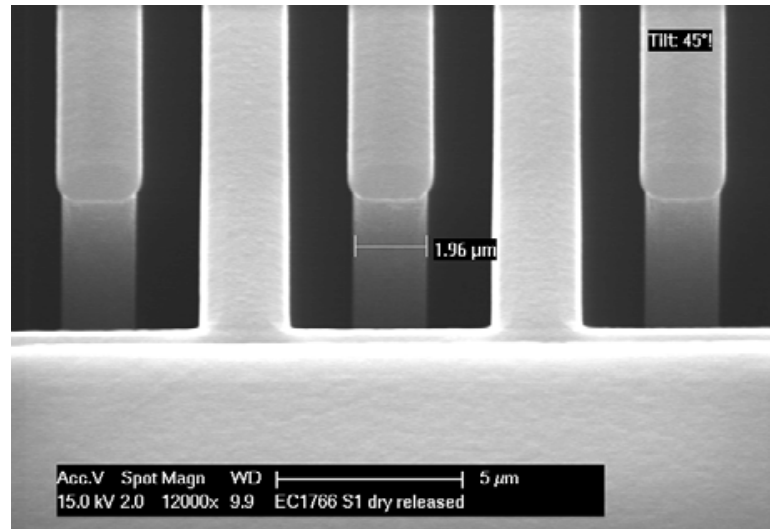

Figure 17. The width of combdrive fingers structure has dimension variation close to the designed value, that is $2 \mu \mathrm{m}$.

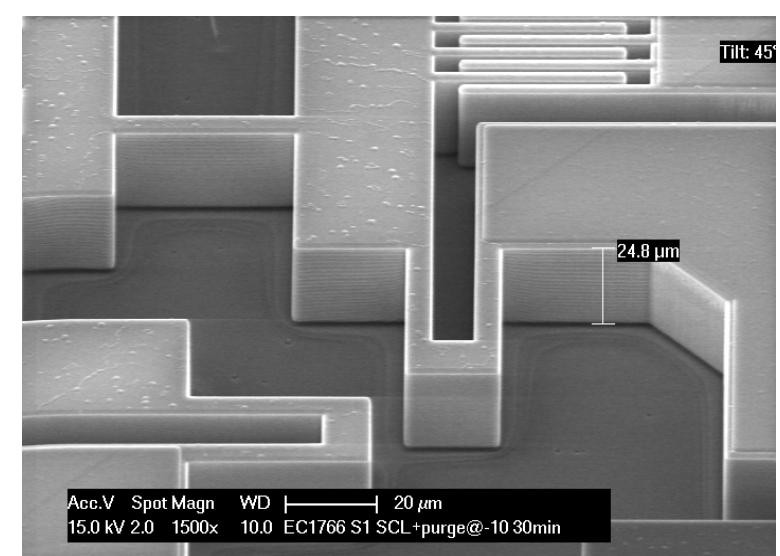

(a)

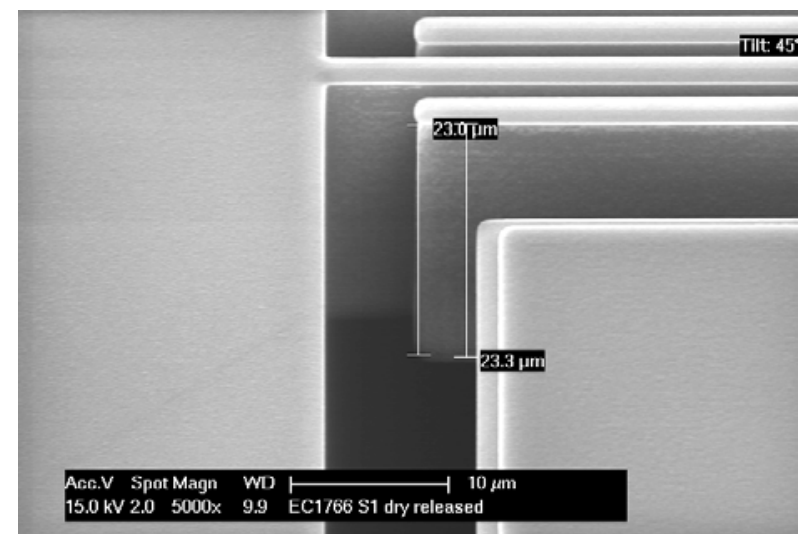

(b)

Figure 18. The variation of the suspended silicon structure thickness in combdrive.

Figure 18 shows the size variation that can be tolerated on the structure thickness of the suspended gyroscope mass. 


\section{SEM image for fabrication failure evaluation}

The evaluation of the failure of critical fabrication step process is important to be known for evaluating the fabrication steps in particular, and for the evaluating the overall design in general. Figure 19 and 20 are SEM images showing the failure of the fabrication process.

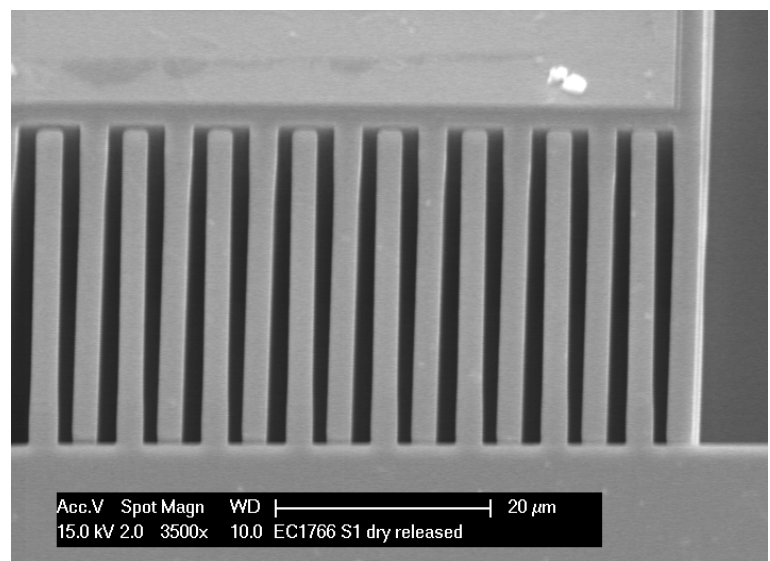

Figure 19. Stiction problems in the failure of the sacrificial layer releasing.

Figure 19 shows the stiction problem occurred when sacrificial layer releasing etch was done. Here, the failure occurred due to the thin sacrificial silicon oxide layer for the releasing etch of the frontside opening. Frontside opening process cannot be done for the standard SOI wafer.

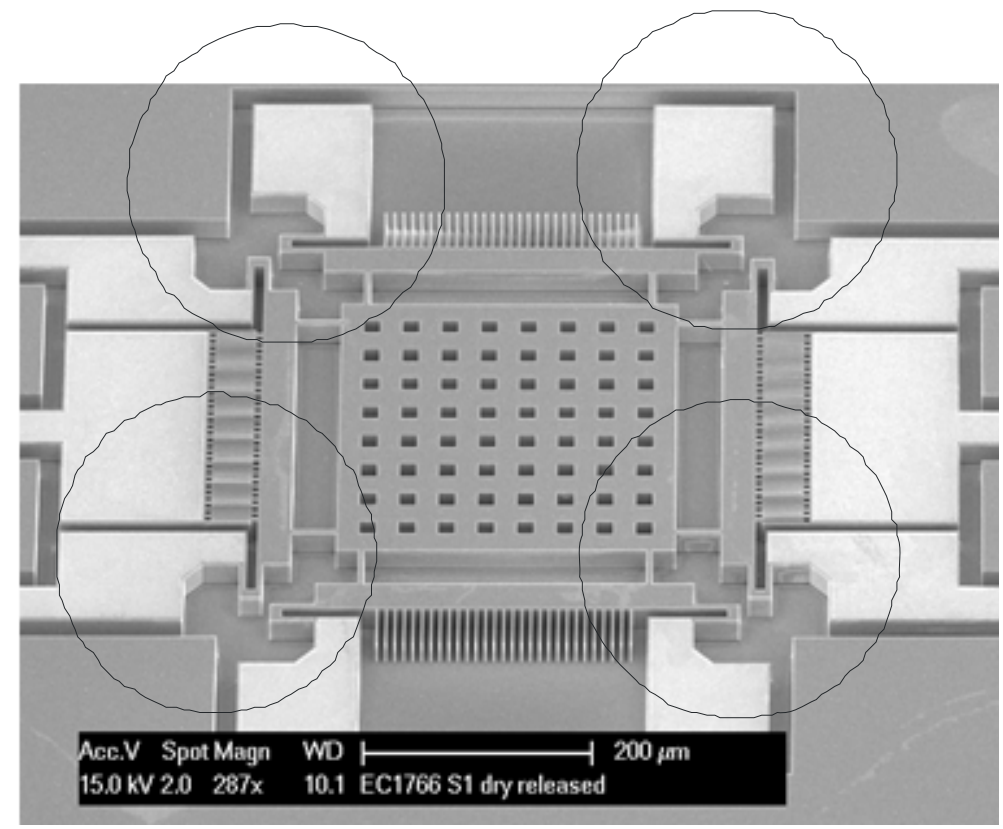

Figure 20. Anchor dimension problem in the failure of the sacrificial layer releasing process.

Figure 20 shows the anchor are detached from the substrate when sacrificial layer releasing etch was done. Here, the anchor dimensions are too small for the releasing etch of the backside opening or the backside opening process was done too long than it should, so it is overetch. 
Tris Dewi Indraswati, et al.

SEM images for the visual test of the resulting physical gyroscope

Next are some examples from the visual test of resulting physical gyroscopes. Figure 21 shows the gyroscope with release holes, which the releasing of the sacrificial layer from the backside of the wafer, seen from above (a) and bottom (b), respectively.

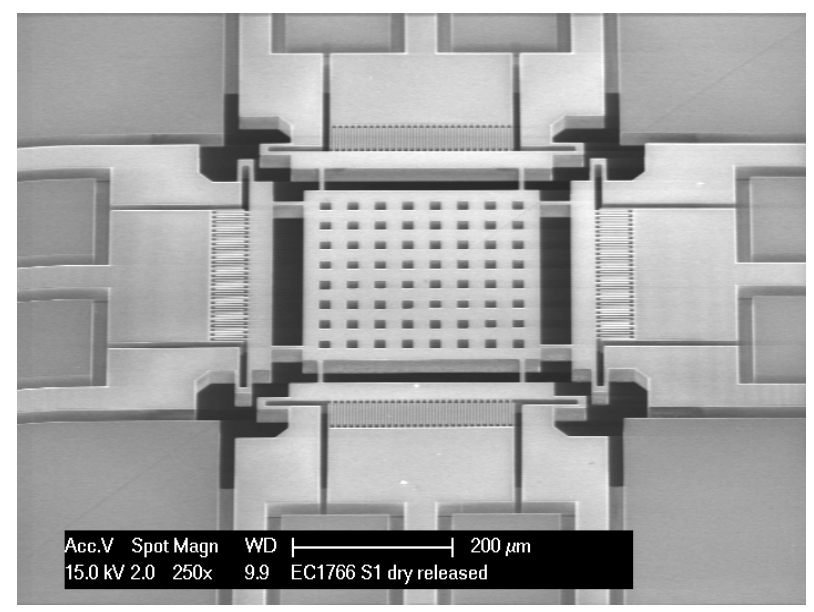

(a).

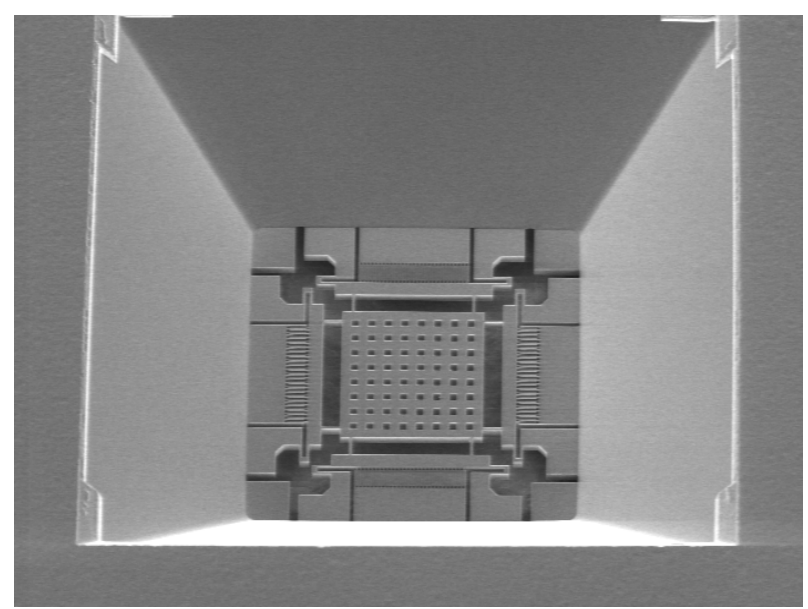

(b).

Figure 21. Gyroscope with proof mass having release holes which the sacrificial layer releasing from the backside, without insulation layer of metal pad, seen from above (a) and bottom (b), respectively.

SEM images for geometry dimension measurement

The following are some examples of SEM images which used to measure the physical dimensions of the resulting gyroscope. Figure 23 shows the dimensions of U-suspension and figure 24 shows the dimensions of $\mathrm{H}$-suspension. The dimension size in one batch processing can vary within acceptable tolerances.

Figure 22 shows the gyroscope having proof mass without release holes, which the releasing of the sacrificial layer from the backside of the wafer, seen from above (a) and bottom (b), respectively. 


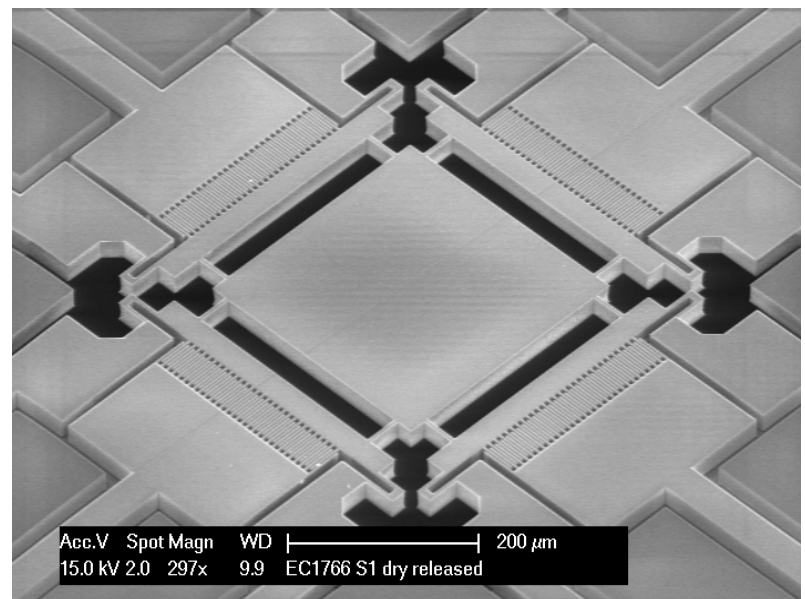

(a).

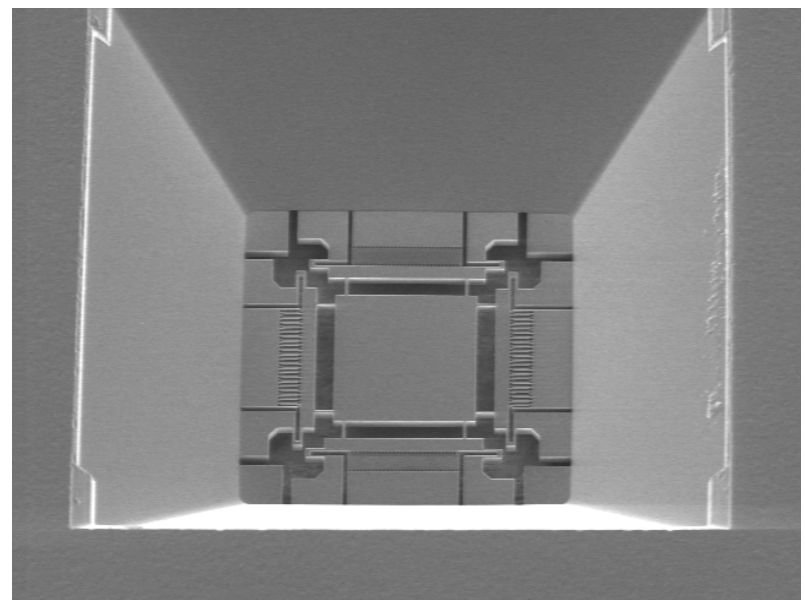

(b).

Figure 22. The gyroscope having proof mass without release holes, which the releasing of the sacrificial layer from the backside of the wafer, without insulation layer of metal pad, seen from above (a) and bottom (b), respectively.

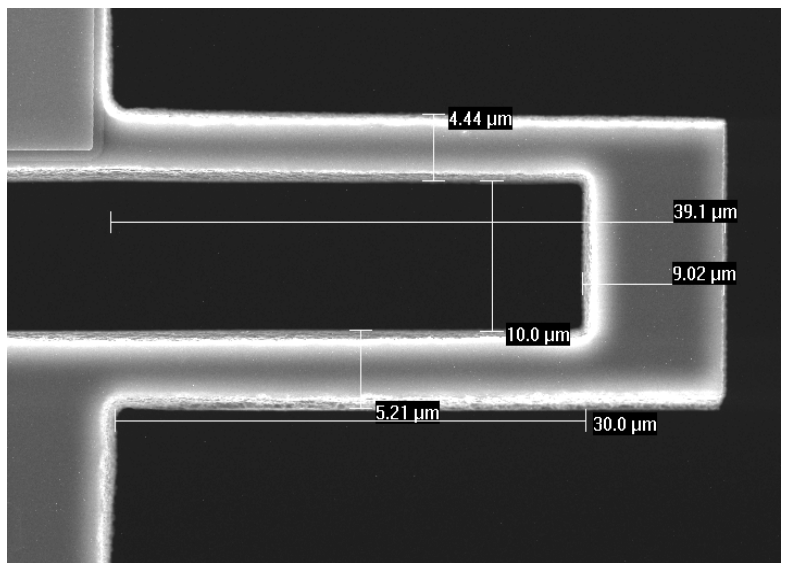

Figure 23. The implementation result of U-suspension in gyroscope with resonance frequency of $8.3 \mathrm{kHz}$. 
Tris Dewi Indraswati, et al.

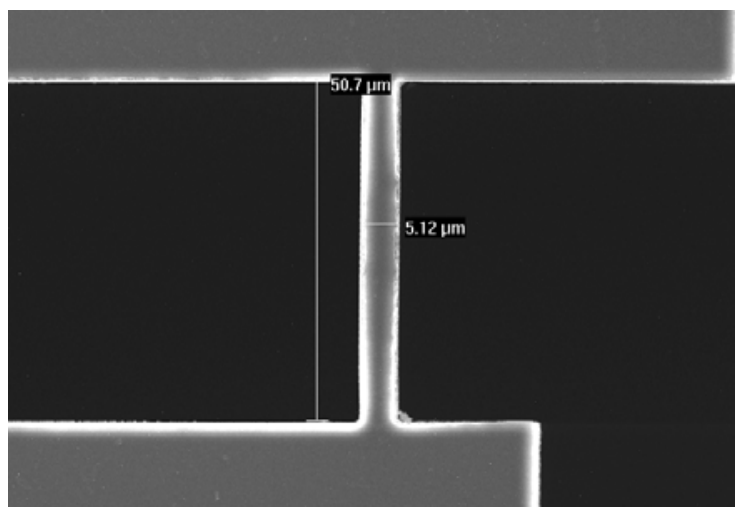

Figure 24. The implementation result of H-suspension in gyroscope with resonance frequency of $8.3 \mathrm{kHz}$.

B. Visually Checking of the Physical Shape and Wiring Checking on the Packaged Gyroscope.

Physical checks on gyroscopes include visually checking of the physical form and wiring checking on the packaged gyroscope. For visually checking of the physical form, it needs the microscope equipment which can enlarge the captured image at least 200 times to see the macro shape of the gyroscope obtained after the fabrication process. To get the visually detail of the separate combdrive fingers, one required the microscope with magnification of at least 500 times. Because the existing microscopes of the Device and IC Processing Laboratory ITB can only magnify 200 times maximum, then some modifications on the microscope were made.

\section{The modifications of probe microscope in Device and IC Processing Laboratory ITB.}

The modification is performed by the addition of a digital camera holder plus digital camera and the addition of a web camera, as shown in Figure 25. The addition of a web camera on the probe microscope has benefits that the pictures captured can be directly sent, displayed and saved on the laptop. In addition to this, some kind of camera holder was made and using the web camera with a higher version to sharpen the pictures obtained, and also the special chip holder and probes were made (Figure 26). Figure 27 shows the difference images obtained with a digital camera (a), web cameras (b), and the dino microscope (c).
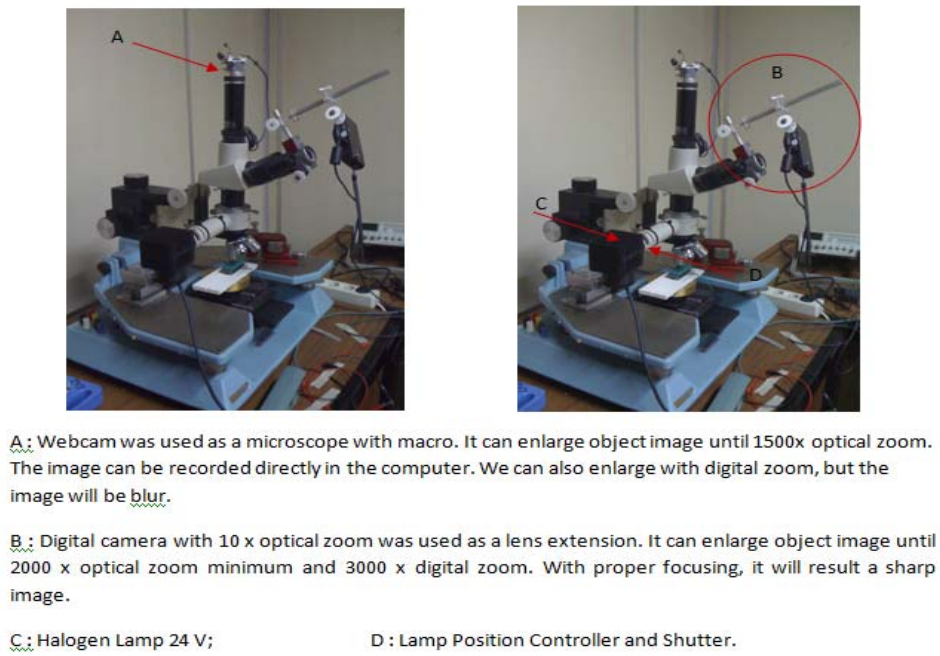

Figure 25. The modification of probe microscope in Device and IC Processing Laboratory in order to get the image magnification enough for checking the MEMS structure in the gyroscope die. 
Visually checking of the physical gyroscope shape.

The gyroscopes yield from the fabrication was packaged in two kind of packaging, namely 16-pins chip package and 40-pins chip package (Figure 28). These gyroscopes were visually checked using probe microscope. The results show that some of gyroscopes on 16-pins chip were damaged, among others, cracks in the U-suspension and imperfections on the proof mass and comb drives.

Wiring checking in gyroscope die, was conducted by measuring the resistance between the pad in the gyroscope die and resistance between pins on the packaged gyroscope die.

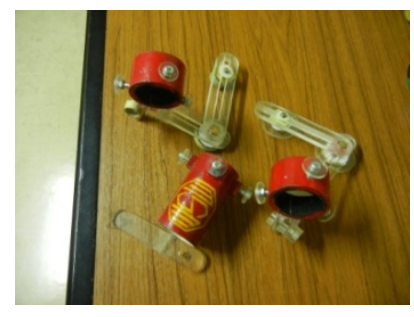

(a).

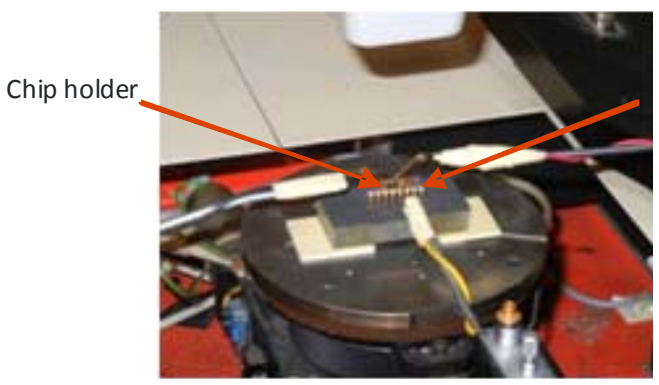

(d).

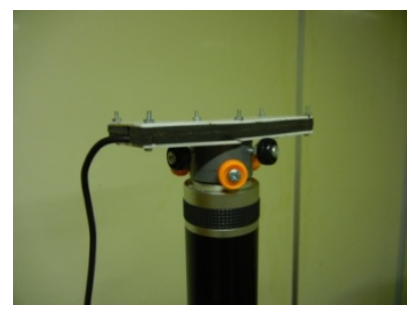

(c).

Probe modification

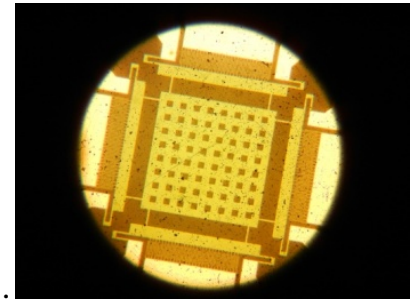

(a).

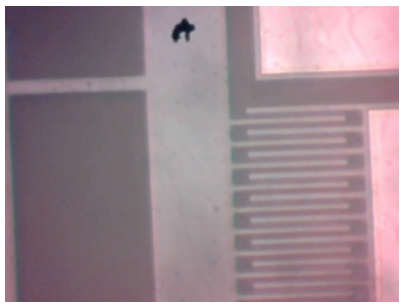

(b).

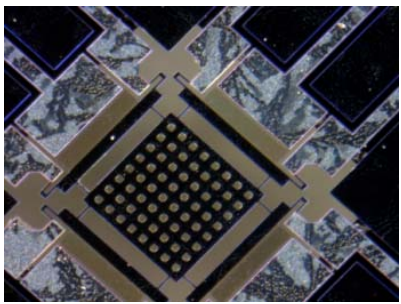

(c).

Figure 27. The different image obtained with digital camera (a), web camera (b), and dino microscope (c). 
Tris Dewi Indraswati, et al.

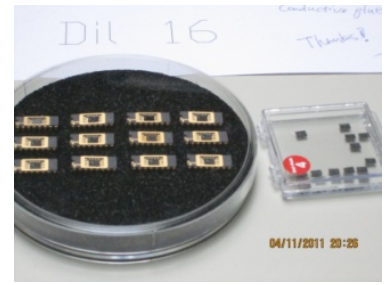

(a).

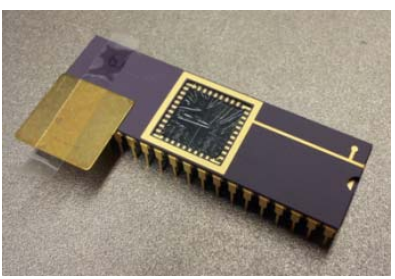

(b).

Figure 28. Gyroscope chips in 16-pins chip package (a) and in 40-pins chip package.

Table 1. Resistance Measurement In 40-PINS Chip Package For Preparation of Bonding and Packaging

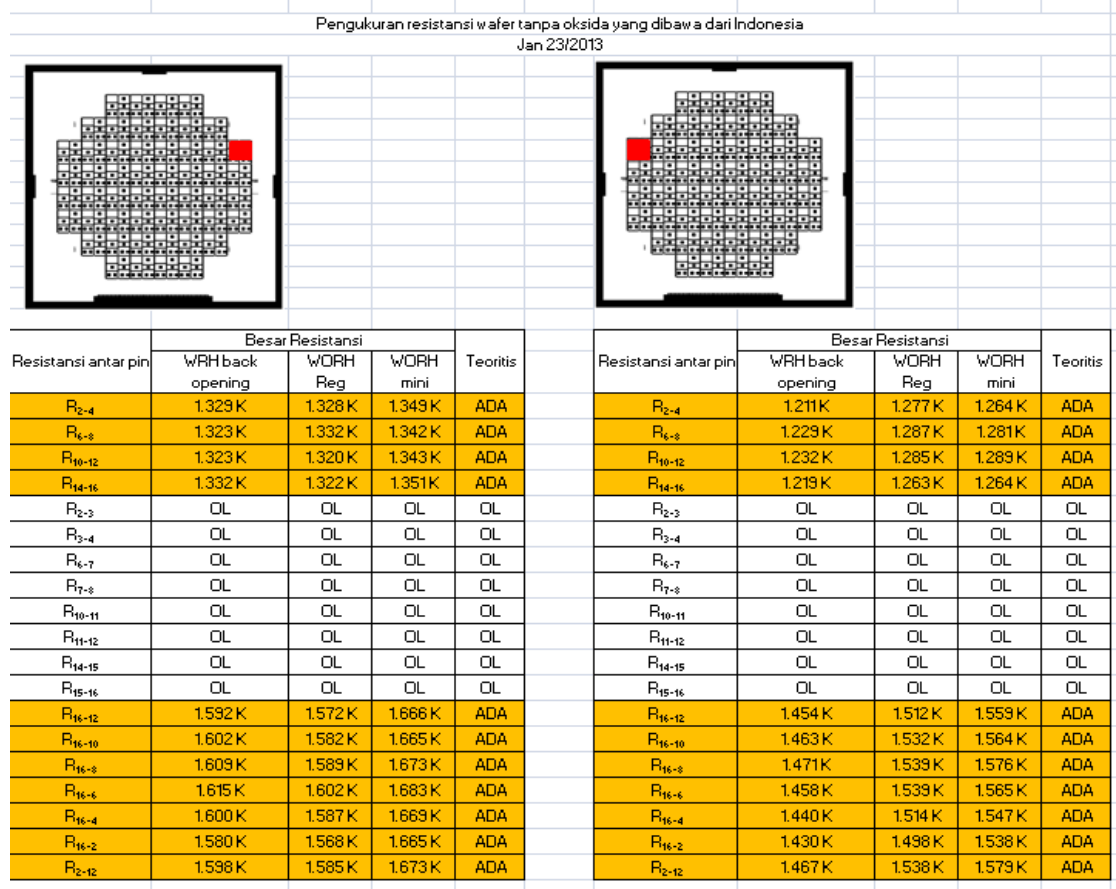

\section{Stiction Problems Checking on the Gyroscope After Fabrication}

After the fabrication process, sometimes there are stiction problems in MEMS devices. This problem occurs because of the surface force (van der Waals, hydrogen bonding, etc.) is much more powerful than the bulk force (spring restoring force, gravity) or formed meniscus during drying step draw the suspended structure to the substrate. Generally it occurs in MEMS structures that are mechanics or have a small gap (e.g. resonator beam). The solution is to avoid the occurrence of MEMS meniscus during drying step in supercritical $\mathrm{CO}_{2}$, where the gas and liquid phases cannot be distinguished; releasing etch with acid vapour or plasma; coat the surface with non-sticking layer.

The stiction problems that occur after the fabrication process can be seen with the profile projector and SEM (Fig. 29). This figure shows that the gyroscope with release holes which the releasing of the sacrificial layer from the frontside of the wafer is not successful. Gyroscope cannot be released from the substrate. The oxide layer between the substrate and the MEMS structure is too thin for the method of front opening, resulting in stiction problems. 


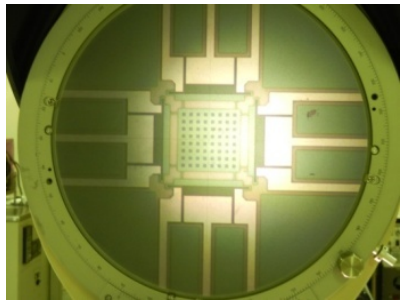

(a).

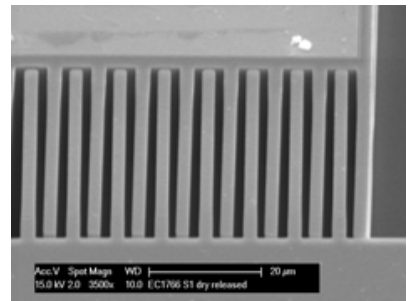

(b).

Figure 29. Stiction problems in gyroscope with release holes which the releasing of the sacrificial layer from the frontside of the wafer.

\section{Vibration Checking on the Gyroscope Mass-Spring Systems}

Vibration testing on the gyroscope mass-spring system is conducted to prove that the massspring system really suspended over the substrate, so that it can vibrate. Several experiments have been carried out with various methods. Some methods did not work as expected. The method gives results is to provide excitation in the form of an acoustic wave with adjustable signal amplitude and frequency. Acoustic wave is propagated from the speaker to the gyroscope via the acoustic table stand (Figure 30). With this method, the mass-spring system looks vibrate and the vibration follow the given amplitude and frequency control. The movement of the gyroscope can be visually seen using web camera set to video recording. Unfortunately, measurements of the quantitative magnitude have not been performed yet.
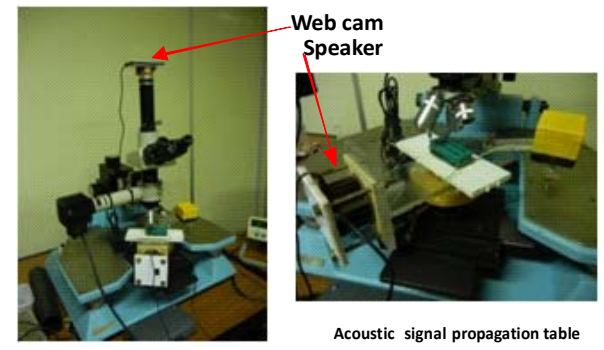

Acoustic signal propagation table

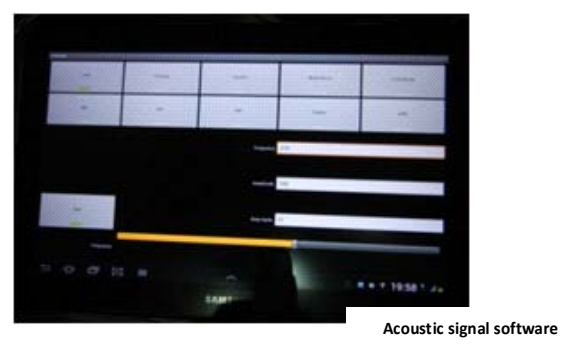

Figure 30. Vibration generation in gyroscope with acoustic excitation method.

\section{Conclusions}

The following are the conclusion from this experiment: Gyroscope with and without release hole can be excellently fabricated using the standard SOI wafer with $400 \mathrm{~nm}$ silicon oxide as the sacrificial layer, using the combination of MEMS bulk and surface technology with DRIE etching techniques on the surface and suspended structure formation using anisotropic etching on the backside of the standard SOI wafer. The fabrication methods can ensure the suspended structure formation, although the sacrificial layer is thin. It had been proven that this methods can remove the curl out of plane problem due to stress gradient. With the modification of the gyroscope fabrication process using standard SOI wafer with $400 \mathrm{~nm}$ silicon dioxide, the suspended structure without release hole can be made, so the mass of the proof mass can be 
increased. Thus, the gyroscope sensitivity is also increased. It had been proven that the capacitance structure made of single crystal silicon with high impurity can increase the capacitance value, thus the gyroscope sensitivity is also increased. The capacitance value can be boost up by increasing ratio aspect with the addition of epitaxial single crystal silicon layer. The fabrication process is relatively simple using 3 mask, so the production cost is reduced. The production cost increase with the addition of the mask number and the more complex fabrication process.

\section{Acknowledgment}

The author would like to thank the Directorate General of Higher Education Ministry of Education and Culture of Indonesia for the funding provided for this research through the Doctor Research Grant Program in 2011 and the S3-Sandwich Grant Program in 2011. Prof.Dr. Adrian Venema introduced the author of this paper to Prof.dr. P.M.Sarro, Head of the Electronic Components, Technology and Materials Laboratory (DIMES) Delft University of Technology, The Netherlands. Prof. Sarro allowed the author to fabricate the studied gyroscope in DIMES and last but not least, Dr. Jia Wei who helps the author in the gyroscope fabrication process.

\section{References}

[1] Nasiri, Steven, "A Critical Review of MEMS Gyroscope Technology and Commercialization Status”, InvenSense.com

[2] Sensors \& Transducers e-Digest, Vol. 67, Issue 5, May 2006: Sensors Market Trends (ISSN 1726- 5479)

[3] Qu, Hongwei, Fang, Deyou, Sadat, Anwar, Yuan,Peter, dan Xie,Huikai, "High Resolution Integrated Micro-Gyroscope for Space Applications", proceedings of the $41^{\text {st }}$ Space Congress, April 27-30, 2004, Cape Canaveral, Florida.

[4] Xie, Huikai, "Gyroscope and Micromirror Design Using Vertical-Axis CMOS-MEMS Actuation and Sensing", Doctoral Degree Dissertation in Electrical and Computer Engineering, Carnegie Institute of Technology of The Carnegie Mellon University, 2002.

[5] Acar, Cenk, "Robust Micromachined Vibratory Gyroscope", Doctoral Degree Dissertation in Mechanical and Aerospace Engineering, University of California, Irvine, 2004.

[6] “Advanced Technology in Surgery”, Curr Probl Surg 2002; 39:7 33-832, August 2002.

[7] Luo, H., Zhu, X., Lakwadala, H., Carley, L. R., and Fedder, G. K., ”A Copper CMOS MEMS Z-Axis Gyroscope”, 0-7803-7815-2/02/\$10.00 @2002 IEEE.

[8] Ayazi, Farrokh and Najafi, Khalil, "A HARPSS Polysilicon Vibrating Ring Gyroscope”, Journal of Microelectromechanical Systems, Vol. 10, No. 2, June 2001.

[9] Alper, Said Emre, Silay, Kanber Mithat, and Akin, Tayfun, "A Low-Cost Rate-Grade Nickel Microgyroscope”, Sensors and Actuators A 132 (2006) 171-181, 2006.

[10] Satava, Richard, "How the Future of Surgery Changing: Robotics, Telesurgery, Surgical Simulators and other Advanced Technologies", Robotics, Telesurgery, \& Surgical Simulators - Miguel Caiznos, May, 2006.

[11] Taylor, R.H., Menciassi, A., Fichtinger, G., and Dario, P., "Medical Robotics and Computer-Integrated Surgery", Part F: Field and Service Robotics, p.1199-1222.

[12] Woodman, Oliver J., “An Introduction to Inertial Navigation”, Technical Report, No. 696, Computer Laboratory, University of Cambridge, Augusts 2007.

[13] Saukoski, Mikko, "System and Circuit Design for A Capacitive MEMS Gyroscope", Doctoral Dissertation, Helsinki University Of Technology, April 18, 2008.

[14] Tsai, Nan-Chyuan, and Sue, Chung-Yang, "Fabrication and Analysis of A MicroMachined Tri-Axis Gyroscope", Journal of Micromechanics and Microengineering 18 (2008) 115014 (14pp), IOP Publishing Ltd. Online at stacks.iop.org/JMM/18/115014. 
[15] Kranz, Michael, "Design, Simulation, and Implementation of Two Novel Micromechanical Vibratory-Rate Gyroscopes”, Thesis, Department of Electrical and Computer Engineering, Carnegie Mellon University, May 1998.

[16] Shkel, Andrei M,. Acar, Cenk, and Painter, Chris, "Two Types of Micromachined Vibratory Gyroscopes”, 0-7803-9056-3/05/\$20.00 (C) 2005 IEEE.

[17] Names unknown, "Basic Principles of Inertial Navigation”, ppt presentation in Seminar on Inertial Navigation Systems in Tampere University of Technology, 2007.

[18] Wei, Jia, "Sillicon MEMS for Detection of Liquid and Solid Fronts", Doctoral Dissertation in Electrical Engineering, Delft University of Technology, 2010.

[19] Xie, Huikai and Fedder, Gary K., “A DRIE CMOS-MEMS Gyroscope”, 0-7803-74541/02/\$17.00@2002 IEEE.

[20] Xie, Huikai and Fedder, Gary K., “Integrated Microelectromechanical Gyroscopes”, Journal of Aerospace Engineering (C) ASCE, p.65-75, April 2003.

[21] Alper, Said Emre, Azgin, Kivanc, and Akin, Tayfun, "A High Performance Silicon-onInsulator MEMS Gyroscope Operating at Atmospheric Pressure”, Sensors and Actuators A 135 (2007) 34-42, 2007.

[22] Shkel, Andrei M. and Zeng, Fan-Gang, "An Electronic Prosthesis Mimicking The Dynamic Vestibular Function”, Audiology and Neurotology Journal, 2006.

[23] Acar, Cenk and Shkel, Andrei M., "MEMS Vibratory Gyroscopes: Structural Approaches to Improve Robustness", Springer, 2009.

[24] Venema, Adrian F. N., "Notes on the Modelling of a Translational Vibratory Z-Axis Gyroscope”, Sekolah Teknik Elektro dan Informatika, Institut Teknologi Bandung, April 2009, revised on March 2013, internal publication, unpublished.

[25] Gardner, Julian K., Varadan,Vijay K., and Awadelkarim,Osama O., ”Microsensors, MEMS, and Smart Devices”, John Wiley \& Sons, second edition, 2005.

[26] Allen, James J., ”Micro Electro Mechanical Systems Design”, Taylor and Francis Group, CRC, 2005.

[27] Maluf, Nadim, and Williams, Kirt, ”An Introduction to Microelectromechanical Systems Engineering”, Artech House, Inc., second edition, 2004.

[28] Osiander, Robert, Darrin, M. A. G., and Champion, John L., ’MEMS and Microstructures in Aerospace Applications", Taylor and Francis Group, CRC, New York, 2006.

[29] Senturia, Stephen D., ”Microsystem Design”, Kluwer Academic Publishers, Dordrecht, 2001.

[30] Kranz, M., Hudson, T., Ashley, P., Ruffin, P., Burgett, S., Temmen, M., and Tuck, J., ”A Single Layer Silicon-on-Insulator MEMS Gyroscope for Wide Dynamic Range and Harsh Environment Applications”, MEMS Components and Applications for Industry, Automobiles, Aerospace, and Communication, Henry Helvajian, Siegfried W. Janson, Franz Lärmer, Editors, Proceedings of SPIE Vol. 4559 (2001).

[31] Sharma, Mrigank, ”Design and Modeling of Advanced Gyroscopes”, Master of Applied Science Thesis in Faculty of Graduate Studies Electrical and Computer Engineering, University of British Columbia, Vancouver, Canada, September 2008.

[32] Xie, Huikai, and Fedder, Gary K., "Design and Fabrication of A Bulk Si CMOS-MEMS Gyroscope”, JMEMS, 2001.

[33] Watanabe, Y., Mitsui, T., Mineta, T., Matsu, Y., Okada, K., “SOI Micromachined 5-axis Motion Sensor Using Resonant Electrostatic Drive and Non-Resonant Capacitive Detection Mode”, Sensors and Actuators A 130 - 131, 2006, p.116-123.

[34] Trusov A.A., Shkel A.M., "Capacitive Detection in Resonant MEMS with Arbitrary Amplitude of Motion”, Mechanical and Aerospace Engineering Department, Micro Systems Laboratory EG2110, University of California, 2007.

[35] Trusov A. A., Shkel A.M., "A Novel Capacitive Detection Scheme With Inherent SelfCalibration”, Journal of Microelectromechanical Systems, Vol.16, No.6 , 2007. 
[36] Trusov A. A., "Investigation of Factors Affecting Bias Stability and Scale Factor Drifts in Coriolis Vibratory MEMS Gyroscopes”, PhD Dissertation, University Of California, 2009.

[37] Depo, Nataniel, "Perancangan dan Implementasi Rangkaian Pengkondisi dan Pemroses Sinyal MEMS-Giroskop”, Bachelor degree Final Project, Sekolah Teknik Elektro dan Informatika, Institut Teknologi Bandung, 2011.

[38] Cowen, A., Hames, G., Monk, DeMaul, Wilcenski, S., Hardy, B., "SOIMUMPS Design Hand book : a MUMPs® process ", MEMSCAP Inc., Revision 7.0, Copyright (C) 20022011 by MEMSCAP Inc.

[39] http://www.unmanned.co.uk/unmanned-vehicles-news/unmanned-aerial-vehicles-uavnews/little-rotary-uavs-are-big-help-to-mounties/

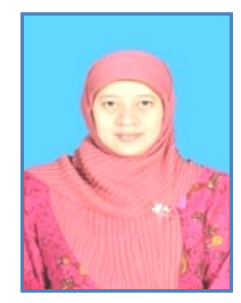

Tris Dewi Indraswati was born in Indonesia, November 2, 1969. I received my B.Eng degree in electronics in 1994 from Bandung Institute of Technology and M.Eng degree also in electronics from the same Institute. I hold my Dr in Electronics from Bandung Institute of Technology (ITB), Indonesia in 2013. Since 1995, I was a lecturer in Electrical Engineering Department, Institut Teknologi Indonesia. I have work on research in gyroscope technology since 2001. My current interest are on MicroElectro Mechanical Systems.

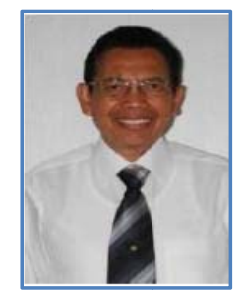

Adang Suwandi Ahmad was born in Lembang, West Java, Indonesia on November 3, 1948. He graduated from his Bachelor degree program in Electrical Engineering ITB; his Master and Doctoral program from USTL, Montpellier, France. His research interests includes Systems Cognitive Instrumentation. He is a member of several professional organizations: SPIE, IEEE Society of Devices \& Aero-space Electronics and Societe Francaise DuVide. He has won several Education and Research awards including: The best Researcher, Research Grant Seminar Donation Research Compete VIII Directorate General Higher Education, National Award of DIKTI DEPDIKNAS in 2002; Satyalancana DWIDYA SISTHA, TNI, Defense Education in 2000; and Satyalancana Karya Satya XXX (30 years), ITB for Education Dedication in 2009.

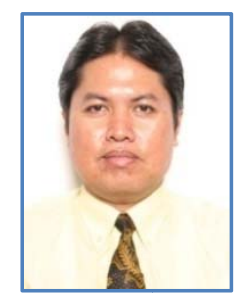

Irman Idris was born in Indonesia, January 29, 1966. He received Bachelor of Engineering degree in Electronics from Bandung Institute of Technology in 1989. Then, he received Master and Doctor of Engineering degrees in Physical Electronics from Tokyo Institute of Technology, Japan in 1996 and 1999, respectively. Since 1990, he has been a lecturer at School of Electrical Engineering and Informatics, as well as a senior researcher at Center for Microelectronics, Bandung Institute of Technology. His current research interests are development of MEMS sensors, micro- and nano-devices, and nano-materials for agriculture, medical, and navigation applications. 


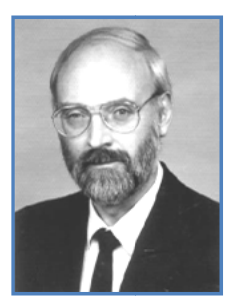

Adrian Venema was born in 1932 in Cimahi (West-Java, Indonesia) and obtained his scientific degrees from the Delft University of Technology, The Netherlands. He lectured for many years in semiconductor physics and modern sensor technology. Modern sensor technology implies heavily on the knowledge of physics and on the miniaturization of the considered sensors.

With his co-workers in the Micro-Acoustic Devices Group (Delft University of Technology), research was performed on acoustic-wave based devices to be implemented in silicon technology as chips, for example sensors for (bio)chemical purposes and fluid dynamics. The results were internationally published in peer-reviewed papers and on conferences.

From 1985 he presented short courses and conference presentations at the Institut Teknologi Bandung (ITB). After retirement in 1997 from the Delft University of Technology, he lectured at ITB mainly in semiconductor physics, data- acquisition systems and modern sensor technology. 